\title{
ANALYTIC FUNCTIONS OVER VALUED FIELDS
}

\section{R. BHASKARAN and V. KARUNAKARAN}

School of Mathematics

Madurai Kamaraj University

Madurai 625021 , INDIA

(Received October 7, 1986 and in revised form June 5, 1987)

ABSTRACT. Let $\mathrm{K}$ be a non-archimedean, non-trivially (rank 1) valued complete field. $B, B^{\circ}$ denote the closed and open unit ball of $K$ respectively. Necessary and sufficient conditions for analytic functions defined on $B, B^{O}$ with values in $K$ to be injective, necessary and sufficient conditions for fixed points, the problem of subordination are studied in this paper.

KEY WORDS AND PHRASES. Non-archimedean valuation, Analytic, Univalent, Subordination, Fixed points.

1980 AMS SUBJECT CLASSIFICATION CODE. 46P05, 32E27, $26 \mathrm{E} 30$.

1. INTRODUCTION.

Let $\mathrm{K}$ be a non-archimedean, non-trivially ( rank 1) valued complete field. $\mathrm{B}, \mathrm{B}^{\mathrm{O}}$ denote respectively the closed and open unit ball of $\mathrm{K}$. In the theory of functions of a single complex variable several deep results are proved using Cauchy's theorem in some form. The present study is aimed at analytic functions defined on $B, B^{\circ}$ with values in $K$. In our case the stronger triangle inequality helps us to prove deeper results with less complications. For example, the local correspondence theorem, Schwarz' lemma, to cite a few. The local correspondence theorem is our starting point for the study of injective analytic functions on B and Lazard's theorem (Theorem 3.20, p.75, [1]), more precisely its consequence is the basis. First we list some results used in the later part. We then take up the problem of finding the necessary and sufficient conditions for analytic functions to be injective (one-to-one), the problem of subordination, conditions for the existence of fixed points etc.

We shall assume throughout this paper that $\mathrm{K}$ is algebraically closed so that the valuation is necessarily dense.

2. PRELIMINARIES.

A function $f: B \rightarrow K$ is said to be analytic if there exists a sequence $\left\{a_{n}\right\}$ of elements in $\mathrm{K}$ such that $f(\lambda)=\sum_{0}^{\infty} a_{n} \lambda^{n}, \lambda \varepsilon B$. Clearly $a_{n} \rightarrow 0, n \rightarrow \infty$ and if $\left\{a_{n}\right\}$ is any such sequence then $f(\lambda)=\sum_{0}^{\infty} a_{n} \lambda^{n}$, defines an analytic function on $B$. Let $A n(B)$ stand for the space of all analytic functions on $B$. If $f \in A n(B)$ then $f$ is bounded and we let $\|f\|=\sup \left|a_{n}\right|$. 
An(B) with this norm is a normed linear space over $K$. If $f(\lambda)=\sum_{D}^{\infty}=a_{n} \lambda^{n}, g(\lambda)=$ $\sum_{0}^{\infty} b_{n} \lambda^{n}$ are in $A n(B)$ then ( $\left.f \cdot g\right)(\lambda)=\sum_{0}^{\infty} c_{n} \lambda^{n}, c_{n}=\sum_{m=0}^{n} a_{m} b_{n-m}$ is also in An(B). Also if $f(\lambda)=\sum_{0}^{\infty} a_{n} \lambda^{n}$ is in $A n(B)$ then $t$ is differentiable in the usual sense and $f^{\prime}(\lambda)=$ $\sum_{1}^{\infty} \mathrm{na}_{n} \lambda^{\mathrm{n}-1}$. We say that $\mathrm{f}(\lambda) \varepsilon \mathrm{An}(\mathrm{B})$ is invertible if there exists $\mathrm{g}(\lambda) \varepsilon$ An $(B)$ such that $f \cdot g=1$. Equivalently $f(\lambda)=\sum_{0}^{\infty} a_{n} \lambda^{n}$ is invertible if and only if $\left|a_{n}\right|<\left|a_{0}\right|$, $\mathrm{n}=1,2, \ldots \ldots$.

A polynomial of the form $\lambda^{m}+\beta_{m-1}{ }^{m-1}+\ldots+\beta_{0}, m \leq N,\left|\beta_{i}\right| \leq 1, i=0,1, \ldots, m-1$, is called a Weierstrass polynomial. Clearly all the roots of the Weierstrass polynomial are in $B$. We state now Lazard's theorem and its consequence without proof.

THEOREM 2.1 (Lazard). Let $f(\lambda)=\sum_{0}^{\infty} a_{n} \lambda^{n}$ be any non-zero analytic function on $B$ and $m=\max \left(i:\left|a_{i}\right|=\|f\|\right)$. Then for every aralytıc function $g$ on $B$ chere exist unique analytic functions $q, r$ such that $r$ is a polynomial of degree less than $m$ and $g=q f+r$. Further $\|g\|=\max (\|q\|\|f\|, \| r i)$.

THEOREM 2.2. Let $\mathrm{f}, \mathrm{m}$ be as in 'Theorem 2.1. Then there exists an invertible element $g$ in $A n(B)$ and elements $\beta_{c}, \beta_{1}, \ldots \beta_{m-1}$ of $K$ such that $\left|\beta_{i}\right| \leq 1, i 0,1 \ldots, m-1$ and $f(\lambda)=$ $\left(\lambda^{m}+\beta_{m-1} \lambda^{m-1}+\ldots+\beta_{0}\right) g(\lambda)$. Further $\beta_{o} \beta_{1}, \ldots, \beta_{m-1}$ and $g(\lambda)$ are unique.

Note: If $f \varepsilon A n(B)$ and $f$ has no zeros in $B$ then $f$ is invertible.

A function $f: B^{\circ} \rightarrow K$ is said to be analytic if there exists a sequence $\left\{a_{n}\right\}$ of elements in $K$ such that $f(\lambda)=\sum_{0}^{\infty} a_{n} \lambda^{n}, \lambda \varepsilon B^{\circ}$. We denote by $A n\left(B^{\circ}\right)$ the space of all analytic functions on $B^{\circ}$. In contrast to the case of $A n(B)$ we note that if $f \varepsilon A^{\circ}\left(B^{\circ}\right)$, then $f$ need not be bounded (choose $\left\{a_{n}\right\}$ in $K$ such that $n-1<\left|a_{n}\right|<n$. Then $f(\lambda)=$ $\sum_{0}^{\infty} a_{n} \lambda^{n}$ defines an analytic function on $B^{\circ}$. By Lemma 6.41, p.235, [1], $\sup \left\{|f(\lambda)|: \lambda \varepsilon B^{\circ}\right\}=$ $\sup \left|a_{n}\right|$. By choice of $\left\{a_{n}\right\}, \sup \left|a_{n}\right|$ is not finite (i.e. $f$ is not bounded). Infact we have $f \in A n\left(B^{\circ}\right)$ if and only if $\lim \sup \left|a_{n}\right|^{1 / n} \leqq 1$, where $f(\lambda)=\sum_{c}^{\infty} a_{n} \lambda^{n}$. For the proofs of the statements made in this section and for more details we refer to [1].

3. MAIN RESULTS.

In this section we first establish the local correspondence theorem. This enables us to normalize the injective functions so that they have the form $\lambda+a_{2} \lambda^{2}+a_{3} \lambda^{3}+\ldots$

THEOREM 3.1 (Local Correspondence). If $f(\lambda) \varepsilon \operatorname{An}(B)$ and $f(\lambda)-w_{0}$ has a zero of order $m$ at $\lambda_{0} \varepsilon B$ then there exists a neighbourhood $N\left(w_{0}\right)$ of $w_{0}$ and a neighbourhood $N\left(\lambda_{0}\right)$ of $\lambda_{0}$ in $B$ such that each value in $N\left(w_{0}\right)$ is assumed exactly $m$ times in the neighbourhood $\mathrm{N}\left(\lambda_{\mathrm{o}}\right)$ at $\mathrm{m}$ distinct points.

PROOF. Let $f \in A n(B)$ and $f(0)=0$ so that 0 is a zero of order $m$. Then $f(\lambda)=$ $\lambda^{m}\left(a_{m}+a_{m+1} \lambda+\ldots\right)$. Let $w \varepsilon k$ be $\operatorname{such}$ that $|w|<\| f l=\sup \left\{\left|a_{i}\right|: i \geq m\right\}$. Then we note that the Weierstrass polynomials for $f$ and $f-w$ are of the same degree (see Theorem 2.2 for the description of the degree of the Weierstrass polynomial). Let $S_{r}(0)$ be a suitable neighbourhood of 0 in which $a_{m}+a_{m+1} \lambda+\ldots$ is invertible. Choose $\alpha \varepsilon K$ 
such that $0<|\alpha|<\min (1 /\|f\|, r, 1)$. Define $g(\lambda)=f(\alpha \lambda) / \alpha^{m}$. $g(\lambda)$ is clearly analytic and $O$ is a zero of order $m$ for $g$. Let $w$ be such that $|w / \alpha m|<\|g\|$, then the Weierstrass polynomial associated with $g$ and $g-w / \alpha^{m}$ have the same degree. Now $g(\lambda)=$ $\lambda^{m}\left(a_{m}+a_{m+1} \lambda+\ldots\right) .\left(a_{m}+a_{m+1} \lambda+\ldots\right)$ is invertible and so $g(\lambda)$ has the required representation as a product of a Weierstrass polynomial and an invertible element of An(B). Hence $\lambda^{m}$ is the Weierstrass polynomial of 8 . This means the Weierstrass polynomial of $\mathbf{g}-w / \alpha^{m}$ is also of degree $\mathrm{m}$. Since the zeros of an analytic function in $B$ are the same as those of the Weierstrass polynomial it follows that there exist $\lambda_{1}, \lambda_{2}$, $\ldots, \lambda_{m}$ in the unit ball $B$ such that $g\left(\lambda_{i}\right)-w / \alpha^{m}=0$. (i.e. $\left.f\left(\alpha \lambda_{i}\right)=w, i=1,2, \ldots, m\right)$. Thus there are $m$ points in the sphere $S_{|\alpha|}(0)$ at which $f$ takes the value w. Thus for any $w$ in $S_{\|g\||\alpha|^{m}}(0)$, we have $m$ points in $S_{|\alpha|}(0)$ at which the value $w$ is assumed by $f$. In order to make them points distinct choose $\alpha$ so that in $S_{|\alpha|}(0), f^{\prime}(\lambda) \neq 0, \lambda \neq 0$. Suppose $f\left(\lambda_{0}\right)=w_{0}$. Define $g(\lambda)=f\left(\lambda+\lambda_{0}\right)-w_{0}$ so that 8 is analytic and $g(0)=0$. If $\lambda_{0}$ is a zero of order $m$ for $f(\lambda)-w_{0}$ then 0 is a zero of order $m$ for $g$. Hence by the above arguments there exist neighbourhoods $V(U), U(0)$ in the range and domain respectively such that each point $w$ in $V(0)$ is taken exactly $m$ times in $U(0)$ by 8 . (i.e. there exist $\lambda_{1}, \lambda_{2}, \ldots, \lambda_{m}$ in $U(0)$ such that $\left.g\left(\lambda_{i}\right)=w, i=1,2, \ldots, m\right)$. Consequently $f\left(\lambda_{i}+\lambda_{0}\right)=w+w_{0}, i=1,2, \ldots, m$. In other words we have neighbourhoods $U\left(\lambda_{0}\right), v\left(w_{0}\right)$ such that each $w$ in $V\left(w_{0}\right)$ is taken exactly $m$ times by $f$ in $U\left(\lambda_{0}\right)$. The proof is complete. COROLLARY 3.2. The above theorem is also true for $f \varepsilon \operatorname{An}\left(\mathrm{B}^{\circ}\right)$.

PROOF. Let $f \varepsilon A n\left(B^{0}\right)$ and $f\left(\lambda_{0}\right)=w_{0}, \lambda_{0} \varepsilon B^{0}$. Choose $\alpha \varepsilon B^{0}$ such that $\left|\lambda_{0}\right|<$ $|\alpha|<1$ (possible by denseness of the valuation) and define $g(\lambda)=f(\alpha \lambda)$. Clearly $8 \bar{\varepsilon} \operatorname{An}(B)$. Now $g\left(\lambda_{0} / \alpha\right)=f\left(\lambda_{0}\right)=w_{0}$, and $\lambda_{0} / \alpha \varepsilon B$. By Theorem 3.1 we have neighbourhoods $N\left(w_{0}\right)$ of $w_{0}$ and $N\left(\lambda_{0} / \alpha\right)$ of $\lambda_{0} / \alpha$ such that if $w \varepsilon N\left(w_{0}\right)$, there are $m$ points $\lambda_{1}, \lambda_{2}, \ldots, \lambda_{m} \varepsilon N\left(\lambda_{o} / \alpha\right)$ and $g\left(\lambda_{i}\right)=w, i=1,2, \ldots, m$. The proof is complete.

Note: From the above result it follows that a one-to-one analytic function cannot have multiple zeros and in particular there is no loss in generality in assuming the normalization $f(0)=0, f^{\prime}(0)=1$ for one-to-one analytic mappings. This will be assumed henceforth.

Now we are in a position to establish the necessary and sufficient conditions for an analytic function to be injective.

THEOREM 3.3. I $\varepsilon \operatorname{An}(B)$ is one-to-one if and only if $\left|a_{i}\right|<1$, $i \geq 2$, where $f(\lambda)=$ $\lambda+a_{2} \lambda^{2}+a_{3} \lambda^{3}+\ldots$.

PROOF. Let $\left|a_{i}\right|<1, i \geq 2$. Then $\left|f\left(\lambda_{1}\right)-f\left(\lambda_{2}\right)\right|=\left|\left(\lambda_{1}-\lambda_{2}\right)+\sum_{2}^{\infty} a_{i}\left(\lambda_{1}^{i}-\lambda \frac{i}{2}\right)\right|=$ $\left|\lambda_{1}-\lambda_{2}\right|$. This means that $\mathrm{f}$ is one-to-one. 
Conversely let $f$ be one-to-one. Since $f(\lambda)=\lambda\left(1+a_{2} \lambda+\ldots\right)$ by Corollary 2.2 it follows that $p(\lambda)=\lambda$ is the associated Weierstrass polynomial and $1+a_{2} \lambda+a_{3} \lambda^{2}+\ldots$ is invertible (has no zeros). Hence $\left|a_{i}\right|<1, i \geqq 2$ (by the equivalent formulation mentioned in section 2).

Note: Let $f \in A n(B)$ be one-to-one. Then $(f-f(0)) / f^{\prime}(0)$ is an isometry.

THEOREM 3.4. Let $f(\lambda)=\lambda+a_{2} \lambda^{2}+a_{3} \lambda^{3}+\ldots \varepsilon$ An( $\left.B^{\circ}\right)$. Then $f$ is one-to-one if and only if $\left|a_{i}\right| \leqq 1, i \geqq 2$.

PROOF. Let $f \in A n\left(B^{\circ}\right)$. We define $f_{\pi} \varepsilon A n(B)$ for $\pi \varepsilon B^{\circ}$, by $f_{\pi}(\lambda)=f(\pi \lambda) / \pi$. Clearly $f_{\pi} \varepsilon \operatorname{An}(B)$. Also if $f$ is one-to-one $f_{\pi}$ is also one-to-one. Note that $f_{\pi}(\lambda)=\lambda+a_{2} \pi \lambda^{2}+a_{3} \pi^{2} \lambda^{3}+\ldots$ Hence if $f$ is one-to-one, by Theorem 3.3 , as $f_{\pi}$ is also one-to-one, $\left|a_{i} \pi^{i-1}\right|<1, i \geq 2$ (i.e. $\left|a_{i}\right|<1 /|\pi|^{i-1}, i \geq 2$ ). Since the valuation is dense we can choose a sequence $\pi_{n} \varepsilon K$ such that $0<\left|\pi_{n}\right|<1$ and $\left|\pi_{n}\right| \rightarrow 1$. Arguing as above we have $\left|a_{i}\right|<1 /\left|\pi_{n}\right|^{i-1}, i \geq 2, n=1,2 \ldots$. This means $\left|a_{i}\right| \leq 1, i \geq 2$.

Again if $\left|a_{i}\right| \leqq 1, i \geq 2$ and if $\lambda_{1}, \lambda_{2} \varepsilon B^{\circ}$, it follows that $\left|f\left(\lambda_{1}\right)-f\left(\lambda_{2}\right)\right|=$ $\left|\left(\lambda_{1}-\lambda_{2}\right)+\sum_{2}^{\infty} a_{i}\left(\lambda_{1}^{i}-\lambda_{2}^{i}\right)=\right| \lambda_{1}-\lambda_{2} \mid$ and hence $f$ is one-to-one.

Note: As before a one-to-one analytic function on $\mathrm{B}^{\mathrm{O}}$ in the normalized form is an isometry.

Let $S\left(S^{\circ}\right)$ denote the class of all normalized functions in $A n(B)\left(A n\left(B^{\circ}\right)\right)$ that are one-to-one. We have the following properties of $\mathrm{S}$ and $\mathrm{S}^{\circ}$.

1. If $f$ belongs to $S$ or $S^{\circ}$ then $\left|f^{\prime}(\lambda)\right|=1$ and so $f^{\prime}(\lambda) \neq 0$.

2. If $f, g$ belong to $S$ or $s^{0}$ then $(f \Theta g)(\lambda)=f(\lambda)+g(\lambda)-\lambda$ belongs to $s$ or $s^{0}$.

3. If $f, g$ belong to $S$ or $S^{\circ}$ then $(f * g)(\lambda)=\lambda+\sum_{2}^{n} a_{i} b_{i} \lambda^{i}$ also belongs to $s$ or $\mathrm{s}^{\circ}$, where $\mathrm{f}=\lambda+\sum_{2}^{\infty} \mathrm{a}_{\mathbf{i}} \lambda^{\mathrm{i}}, \mathrm{g}=\lambda+\sum_{2}^{\infty} \mathrm{b}_{\mathbf{i}} \lambda^{i}$.

4. If $f$ belongs to $S$ or $S^{\circ}$ then $|f(\lambda)|=|\lambda|$, for $\lambda$ in $B$ or $B^{\circ}$.

5. Let $f=\lambda+\sum_{2}^{\infty} a_{i} \lambda^{i}$ belong to $s$ or $s^{0}$. Then $s_{n}=\lambda+\sum_{2}^{\infty} a_{i} \lambda^{i}$ also belongs to $\mathrm{s}$ or $\mathrm{s}^{\circ}$ for $\mathrm{n}=2,3, \ldots$

6. $f$ belongs to $S$ if and only if $\lambda$ is the associated Weierstrass polynomial. What is the corresponding result for $f$ belonging to $\mathrm{s}^{\circ}$ ?

7. If $f$ belongs to $S$ then for every $\gamma_{0} \varepsilon B^{\circ}$, there exists $\lambda_{0}$ in $B$ such that $f\left(\lambda_{0}\right)=\gamma_{0}$. If $f$ belongs to $s^{\circ}$ then $f\left(B^{\circ}\right)=B^{\circ}$.

8. If $f$ and $g$ are in $s^{\circ}$ then $(f \odot g)(\lambda)=f(g(\lambda))$ also belongs to $s^{\circ}$.

Properties 1 to 6 and 8 are easily verified. We shall verify 7 . Let $\mathrm{f} \varepsilon \mathrm{S}$ and $\gamma_{0} \in B^{\circ}$. Now $f, f-\gamma_{0}$ have Weierstrass polynomials of the same degree since they have the same norm. But $\lambda$ is the Weierstrass polynomial of $f$ since $f$ is an element of $s$. Therefore the degree of the Weierstrass polynomial of $f-\gamma_{0}$ is 1 , i.e. there exists a 
unique $\lambda_{0}$ in $B$ such that $f\left(\lambda_{0}\right)=\gamma_{0}$. If $f \in S^{\circ}$ by property $4, f\left(B^{\circ}\right) \subset B^{\circ}$. Let $\gamma_{0} \in B^{0}$. Define $f_{\pi}(\lambda)=f(\pi \lambda)$ where $0<|\pi|<1, \lambda \varepsilon B$. Then $f_{\pi} \varepsilon$ An(B). So by the above argument there exists $\lambda_{1} \varepsilon B$ such that $f_{\pi}\left(\lambda_{1}\right)=\gamma_{0}$, i.e. $f\left(\pi \lambda_{1}\right)=\gamma_{0}$. But $\lambda_{1} \pi \varepsilon B^{\circ}$. In other words $B^{\circ} \subset \mathrm{f}\left(\mathrm{B}^{\circ}\right)$.

4. SUBORDINATION.

Let $f \in A n\left(B^{\circ}\right), g \in S^{\circ}$. If $f\left(b^{\circ}\right) \subset g\left(B^{\circ}\right)$ and $f(0)=g(0)$, we say that $f$ is subordinate to $\mathrm{g}$. We denote this by $\mathrm{f}<\mathrm{g}$ (the same as the classical notation).

In this section we study the following question:

Let $\mathrm{f}<\mathrm{g}$ and $\mathrm{B}_{\mathbf{r}}=\{\alpha:|\alpha| \leq \mathrm{r}\}, 0<\mathrm{r}<1$. Is it true that $\mathbf{f}\left(\mathrm{B}_{\mathbf{r}}\right) \subset \mathbf{g}\left(\mathrm{B}_{\mathbf{r}}\right), 0<\mathrm{r}<1$ ?

The answer to this question is in the affirmative and we establish this through a sequence of auxiliary results. Among these we have an important result viz., the Schwarz lemma which is of independent interest in itself.

LEMMA 4.1. Let $f \in A n(B)$ be such that $|f(\lambda)| \leq 1, \lambda \varepsilon B$ and $f(0)=0$. Then $|f(\lambda)| \leq|\lambda|$ and $\left|f^{\prime}(\lambda)\right| \leq 1, \lambda \varepsilon B$.

PROOF. We know that $f(\lambda)=p(\lambda)_{g}(\lambda)$ by Corollary 2.2 where $p(\lambda)=\lambda^{m}+\beta_{m-1} \lambda^{m-1}+$ $\ldots+\beta_{1} \lambda$ and $g(\lambda)=a_{0}+a_{1} \lambda+\ldots$ are Weierstrass polynomial and the invertible element associated to f. $g(\lambda)$ being invertible we have $|g(\lambda)|=\left|a_{0}\right|, \lambda \varepsilon$ B. Consider the polynomial $p_{1}(\lambda)=\lambda^{m}+\beta_{m-1} \lambda^{m-1}+\ldots+\beta_{1} \lambda-1$. This is again a Weierstrass polynomial and so has all its roots in B ( $K$ being algebraically closed), i.e. there exists $\lambda_{0} \varepsilon \mathrm{K}$ such that $\mathrm{p}\left(\lambda_{0}\right)=1$. Now $1 \geq\left|\mathrm{f}\left(\lambda_{0}\right)\right|=\left|\mathrm{p}\left(\lambda_{0}\right)\right|\left|\mathrm{g}\left(\lambda_{0}\right)\right|=\left|\mathrm{a}_{0}\right|$. Thus $|\mathrm{f}(\lambda)|$ $=|\mathrm{p}(\lambda)| \mathrm{g}(\lambda)|\leq| \lambda|| \mathrm{a}_{\mathrm{o}}|\leq| \lambda \mid$. Again for $\lambda \varepsilon \mathbf{B},\left|\mathrm{f}^{\prime}(\lambda)\right|=\left|\mathrm{p}^{\prime}(\lambda) \mathrm{g}(\lambda)+\mathrm{p}(\lambda) \mathbf{g}^{\prime}(\lambda)\right| \leq$ $\max \left(\left|p^{\prime}(\lambda)_{g}(\lambda)\right|,\left|p(\lambda)_{g^{\prime}}(\lambda)_{\mathbf{g}}(\lambda)\right|\right) \leq 1$.

The proof is now complete.

LEMMA 4.2. If $f \in A n\left(B^{\circ}\right), f(0)=0$ and $|f(\lambda)|<1$, then $|f(\lambda)| \leq|\lambda|$ and $\left|f^{\prime}(\lambda)\right|$ $\leqq 1, \lambda \in \mathrm{B}^{\circ}$.

PROOF. Let $\lambda \in B^{\circ}$ be fixed. Choose $\pi \varepsilon K$ such that $|\lambda|<|\pi|<1$. Define $f_{1}(\alpha)=$ $f(\pi \alpha), \alpha \varepsilon B$. Then $f_{1} \varepsilon A n(B), f_{1}(0)=0$ and $\left|f_{1}(\alpha)\right| \leq 1$. Hence by Lemma 4.1 it follows that $\left|f_{1}(\alpha)\right| \leq|\alpha|$, $\alpha \varepsilon B$. In particular $|f(\lambda)|=\left|f_{1}(\lambda / \pi)\right| \leq|\lambda| /|\pi|$. Choose a sequence $\pi_{n} \varepsilon K$ such that $|\lambda|<\left|\pi_{n}\right|<1$ and $\left|\pi_{n}\right| \rightarrow 1$. Then we have $|f(\lambda)| \leq|\lambda| /\left|\pi_{n}\right|$, $n=1,2, \ldots$ i.e. $|f(\lambda)| \leq|\lambda| . \quad \lambda$ being arbitrary it follows that $|f(\lambda)| \leq|\lambda|, \lambda \varepsilon B^{\circ}$. Again a similar argument as in Lemma 4.1 shows that $\left|f^{\prime}(\lambda)\right| \leq 1, \lambda \varepsilon B^{\circ}$.

NOTE. Lemmas 4.1 and 4.2 are the analogues of the classical Schwarz Lemma.

Solution to the original question.

Let $g(\lambda)=\lambda+a_{2} \lambda^{2}+a_{3} \lambda^{3}+\ldots$. By hypothesis and property 4 we have $|g(\lambda)|=$ $|\lambda|, \lambda \varepsilon B^{\circ}$. Let $\lambda \varepsilon B_{r}$. Then there exists $\gamma \varepsilon B^{\circ}$ such that $f(\lambda)=g(\gamma)$. But $f<g$ implies that $f(0)=g(0)=0$ and $|f(\lambda)|<1$. Therefore by Lemma $4.2|\gamma|=|g(\gamma)|=$ $|f(\lambda)|=|f(\lambda)| \leqq|\lambda| \leqq r$. In other words $f\left(B_{\mathbf{r}}\right) \subset g\left(B_{r}\right)$. 
5. FIXED POINTS.

By a fixed point (as usual) we mean $\lambda \varepsilon B$ or $B^{\circ}$ such that $f(\lambda)=\lambda$ for $f$ belonging to either $A n(B)$ or $A n\left(B^{\circ}\right)$. We give necessary and sutficient conditions for $f$ in $A n(B)$ or $A n\left(B^{\circ}\right)$ to have fixed points.

THEOREM 5.1. Let $f(\lambda)=\lambda+a_{2} \lambda^{2}+\ldots$ belong to $A n(B)$. $f$ has no fixed points in $B$ other than 0 if and only if $\left|a_{i}\right|<\left|a_{2}\right|, i \geq 3$.

PROOF. If $\left|a_{i}\right|<\left|a_{2}\right|, i \geqq 3$ and $\lambda_{0} \neq 0$ is a fixed point for $f$ then it follows that $\left|a_{2}\right|=0$. This means that $\left|a_{i}\right|<\left|a_{2}\right|$ cannot happen. This is a contradiction. Conversely let $f$ have no fixed points other than 0 i.e. $f(\lambda)-\lambda$ has no zeros in $B$ other than 0 . Therefore $a_{2}+a_{3} \lambda+\ldots$ is invertible and so $\left|a_{i}\right|<\left|a_{2}\right|, i \geq 3$. The proof is complete.

As before we can get the following theorem.

THEOREM 5.2. Let $f(\lambda)=\lambda+a_{2} \lambda^{2}+\ldots$ belong to $A n\left(B^{\circ}\right)$ and $f$ be not the identity map. f has no fixed points other than 0 if and only if $\left|a_{i}\right| \leq\left|a_{2}\right|, i \geq 3$.

PROOF. If $\left|a_{i}\right| \leqq\left|a_{2}\right|, i \geqq 3$, arguing as in Theorem 5.1 we have that $f$ has no fixed points in $B^{\circ}$. For the converse choose $\pi_{m} \varepsilon K$ such that $\left|\pi_{m}\right|<1$ and $\left|\pi_{m}\right| \rightarrow 1$ as $m \rightarrow \infty$. Define $f_{m}(\lambda)=f\left(\pi_{m} \lambda\right) / \pi_{m}, \lambda \varepsilon$ B. Now $f_{m}(\lambda)$ belongs to An(B) and has no fixed points in B. Since $f_{m}(\lambda)=\lambda+\sum_{2}^{\infty} a_{i} \pi_{m}^{i-1} \lambda^{i}$, by Theorem 5.1 we have $\left|a_{i} \pi_{m}^{i-1}\right|<\left|a_{2} \pi_{m}\right|, i \geq 3$, i.e. $\left|a_{i}\right|<\left|a_{2}\right| /\left|\pi_{m}\right|^{i-2}, i \geq 3, m=1,2, \ldots$. In other words $\left|a_{i}\right| \leq\left|a_{2}\right|, i \geq 3$. The proof is complete.

\section{ACKNOWLEDGEMENT}

The authors thank the referee for valuable suggestions. Research of this author is supported by a Career Award from the UGC, India.

REFERENCES

1. VAN ROOIJ, A.C.M. Non-archimedan functional analysis, Marcel Dekker, 1978. 


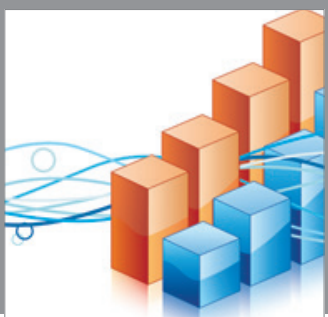

Advances in

Operations Research

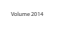

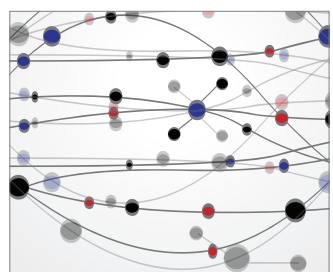

\section{The Scientific} World Journal
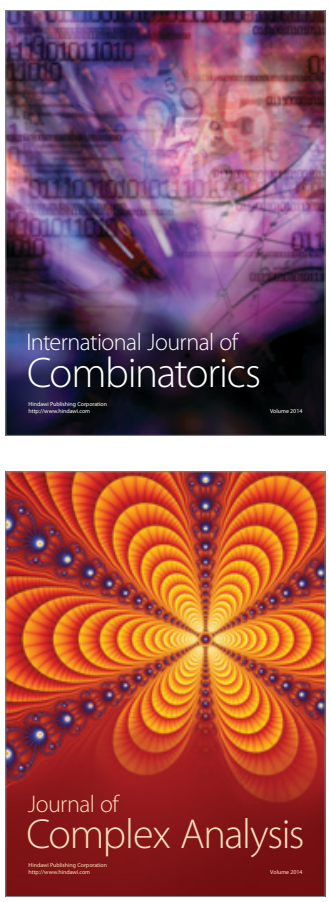

International Journal of

Mathematics and

Mathematical

Sciences
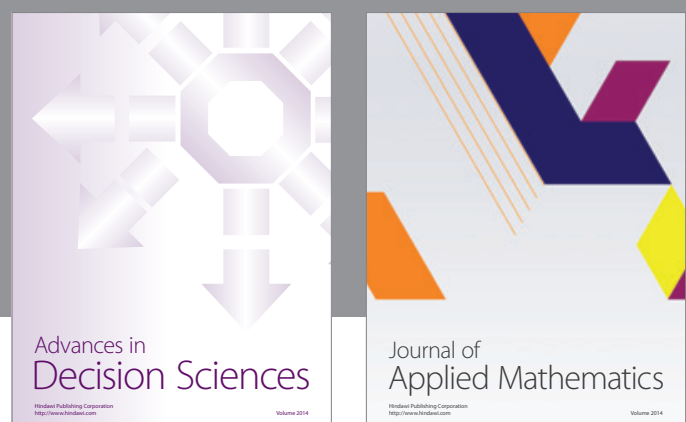

Journal of

Applied Mathematics
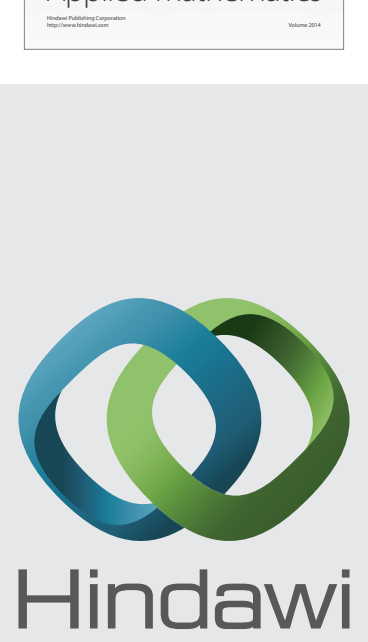

Submit your manuscripts at http://www.hindawi.com
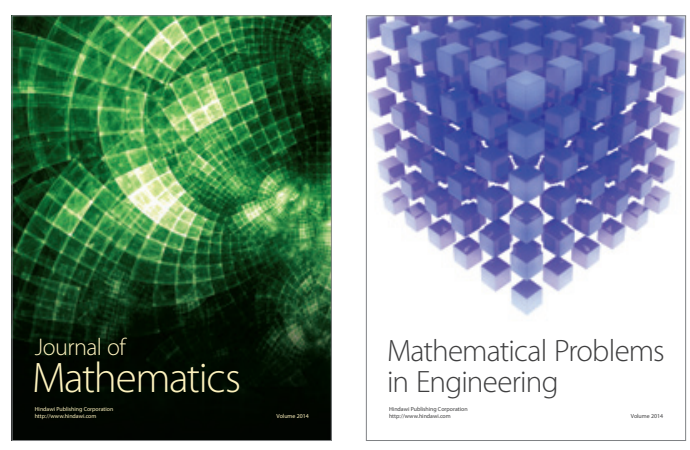

Mathematical Problems in Engineering
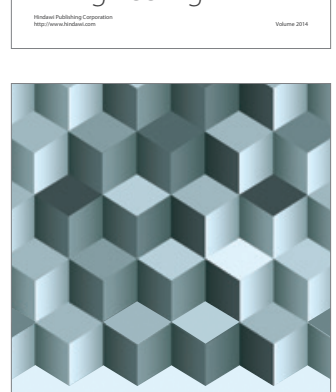

Journal of

Function Spaces
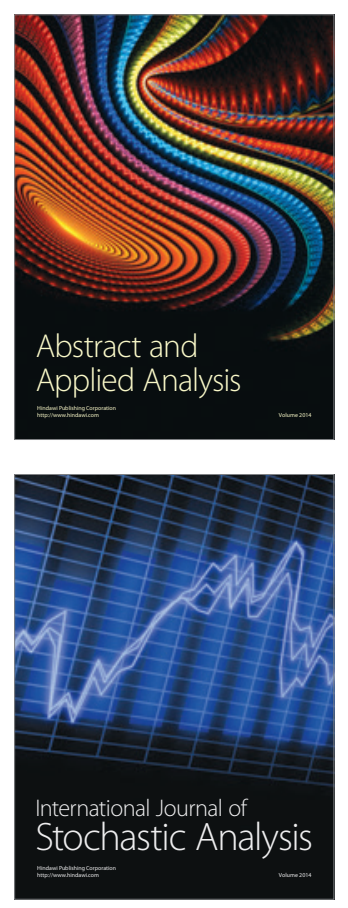

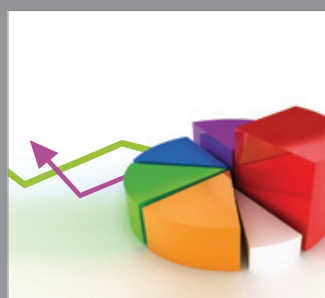

ournal of

Probability and Statistics

Promensencen
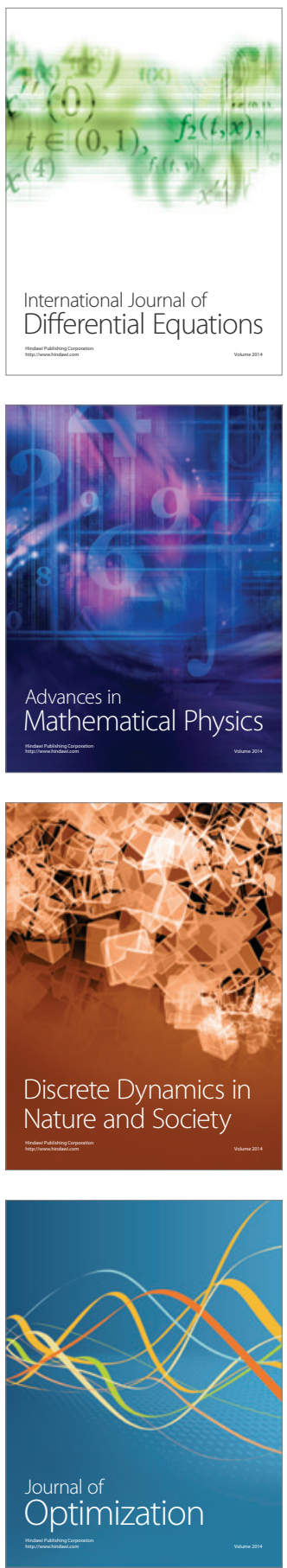